Check for updates

Cite this: RSC Adv., 2019, 9, 29005

\title{
A bio-inspired synthesis of hybrid flavonoids from 2-hydroxychalcone driven by visible light $\uparrow$
}

\author{
Yu-Qi Gao, ${ }^{a}$ Yi Hou, $^{a}$ Liming Zhu, ${ }^{\mathrm{b}}$ Guzhou Chen, ${ }^{a}$ Dongyang $\mathrm{Xu}{ }^{\mathrm{a}}$ \\ Sheng-Yong Zhang, ${ }^{\star c}$ Yupeng $\mathrm{He}^{* \mathrm{~b}}$ and Weiqing Xie (D) *ad
}

\begin{abstract}
A bio-inspired synthesis of hybrid flavonoids from 2-hydroxylchalcone is described. Under the irradiation of 24 W CFL, 2-hydroxychalcone reacts with various nucleophiles to deliver structurally diverse hybrid flavonoids in good to excellent yields in the presence of a catalytic Brønsted acid. Moreover, moderate enantioselectivities could be obtained using a catalytic chiral phosphoric acid via counter anion directed addition. Based on mechanistic studies, the reaction is proposed to proceed via tandem double-bond isomerization/dehydrated cyclization of 2-hydroxychalcone to form a transient flavylium cation, which is in situ captured by nucleophiles to afford hybrid flavonoids.
\end{abstract}

Received 8th September 2019

Accepted 9th September 2019

DOI: 10.1039/c9ra07198a

rsc.li/rsc-advances

which reveals the equilibrium between those flavonoids in solution under the irradiation of UV or sunlight. As shown in Fig. 1, this process is initiated with the $E / Z$ isomerization to deliver $Z$-enone I, which is transferred to flavylium cation II via dehydrative cyclization in the presence of Brønsted acid. The in situ capture of this flavylium cation has been proposed to constitute the key step for the biosynthesis of some type of hybrid flavonoids. ${ }^{7 f}$

Synthesis of hybrid flavonoids via nucleophilic addition to flavylium cation has been extensively explored, ${ }^{8}$ which relies on the high electrophilicity on the $\mathrm{C} 4$ of flavylium cation. ${ }^{9}$ However, those protocols usually need base to act as biosynthetic pathway of flavonoids (Fig. 1, pathway 1), ${ }^{6}$ hybrid flavonoids (e.g. diinsininol ${ }^{5 b}$ ) may be biogenetically generated via nucleophilic addition of flavanone IV to anthocyanidin salt II, which is derived from flavanol III catalyzed by anthocyanidin synthase. Flavanol III is in turn produced from chalcone 2 through a series of enzymepromoted transformations. However, an alternative biosynthetic pathway has also been widely accepted, in which sunlight plays a pivotal role (Fig. 1, pathway 2 ), ${ }^{7}$ In this context, the photochemical interconversion of 2-hydroxychone $\mathbf{1}$ and flavylium salt II has been fully established,

${ }^{a}$ Shaanxi Key Laboratory of Natural Products \& Chemical Biology, College of Chemistry \& Pharmacy, Northwest A\&F University, 22 Xinong Road, Yangling 712100, Shaanxi, China. E-mail: xiewq@nwafu.edu.cn

${ }^{b}$ College of Chemistry, Chemical Engineering and Environmental Engineering, Liaoning Shihua University, Dandong Lu West 1, Fushun 113001, China. E-mail: yupeng.he@lnpu.edu.cn

'Department of Chemistry, Fourth Military Medical University, Xi'an 710032, China. E-mail: syzhang@fmmu.edu.cn

${ }^{d}$ Key Laboratory of Botanical Pesticide R\&D in Shaanxi Province, Yangling, Shaanxi 712100, China

$\dagger$ Electronic supplementary information (ESI) available: General experimental procedures, and spectroscopic date for the all new compounds. See DOI: 10.1039/c9ra07198a

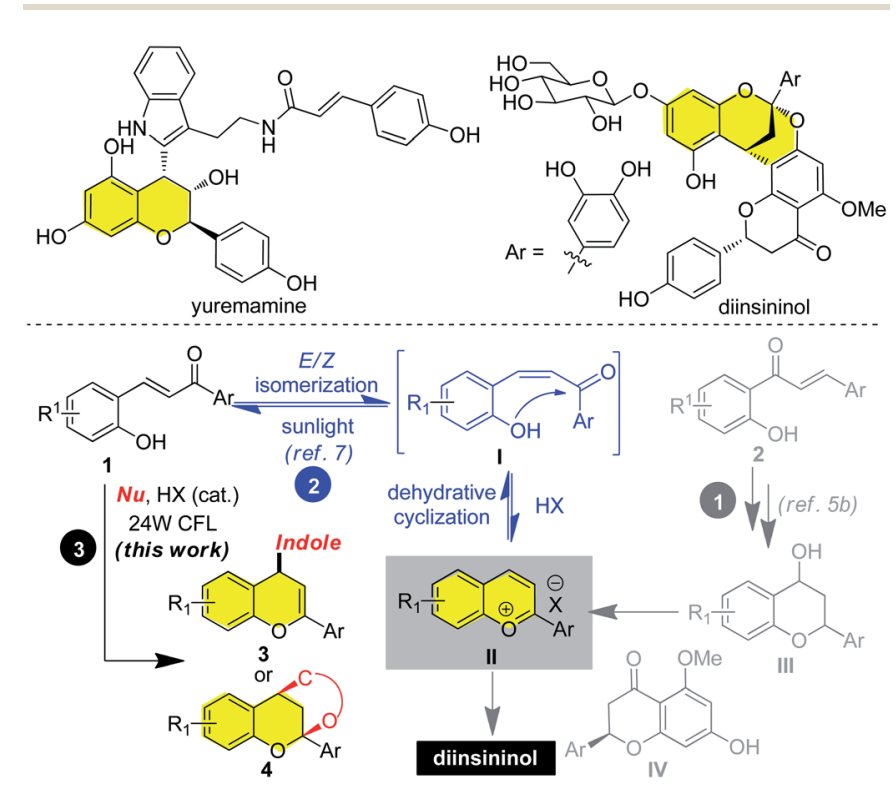

Fig. 1 Previous protocols for the synthesis of hybrid flavonoids and a bio-inspired synthesis of hybrid flavonoids form 2-hydrxoychalcone. 
acid scavenger or heating in protic solvent. Furthermore, the flavylium cation is sensitive to light in solution. ${ }^{7}$ The other commonly employed strategy is the tandem Michael addition/dehydrated cyclization of 2-hydroxylchalcone 1 to deliver flavonoids 3 or polycyclic flavonoids $4 .^{\mathbf{1 0}}$ Different catalysts such as $\mathrm{I}_{2}$, TfOH and $\mathrm{NbCl}_{5}$ have been discovered to be effective for promoting this reaction. However, this strategy is limited by its harsh reaction conditions (heating) or need of precious metal catalysts. Drawn inspiration from the light-driven biosynthetic pathway of hybrid flavonoids (Fig. 1, pathway 3), ${ }^{6,7}$ we proposed that if a suitable nucleophile is exposed to 2-hydroxychalcone 1 in the presence of catalytic Brønsted acid under the irradiation of $24 \mathrm{CFL}$, hybrid flavonoids 3 or 4 could be biomimetically obtained via the in situ capture of the transient flavylium salt. Herein, we would like to describe our preliminary results on this bioinspired synthesis of hybrid flavonoids driven by visible light.

To verify our hypothesis, the reaction of hydroxyl chalcone 1a with indole 5a was initially examined to establish the optimal reaction conditions. To our delight, upon the irradiation of household $24 \mathrm{~W}$ compact fluorescent lamp (CFL) the desired flavonoid 3aa could be obtained in $73 \%$ yield in the presence of phosphoric acid CPA (Table 1, entry 1). Subsequent survey of different kind of Brønsted acids showed that biphenyl phosphate was the most efficient promoter, affording desired product in $98 \%$ yield (Table 1 , entry 2-6 and ESI $\dagger$ ). In sharp contrast, when the reaction was run in the absence of Brønsted acid or in the dark (Table 1, entry 7 and 8), no product could be detected, indicating that the light-driven formation of flavylium from 2-hydroxychalcone might be involved. Subsequently, survey of solvents revealed that THF was the optimal solvent, which

Table 1 Optimization of the reaction conditions ${ }^{a}$

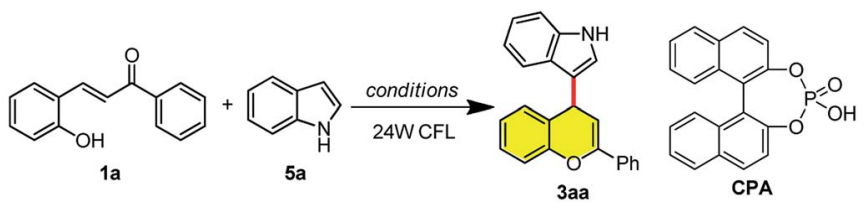

\begin{tabular}{lllll}
\hline Entry & Catalyst & Solvent & Time (h) & Yield $^{b}(\%)$ \\
\hline 1 & CPA & MeCN & 18 & 73 \\
2 & CSA & MeCN & 18 & 42 \\
3 & HCl & MeCN & 18 & 40 \\
4 & Biphenyl phosphate & MeCN & 30 & 98 \\
5 & TsOH & MeCN & 42 & 45 \\
6 & TFA & MeCN & 42 & 43 \\
7 & - & MeCN & 42 & ND \\
$8^{c}$ & Biphenyl phosphate & MeCN & 42 & ND \\
9 & Biphenyl phosphate & THF & 19 & 98
\end{tabular}

${ }^{a}$ Reaction conditions: to a mixture of hydroxyl chalcone $1 \mathrm{a}(0.1 \mathrm{mmol})$ and indole $(0.12 \mathrm{mmol})$ was added solvent $(3 \mathrm{~mL})$. The reaction mixture was stirred with irradiation by household $24 \mathrm{~W}$ compact fluorescent lamp (CFL). ${ }^{b}$ Isolated yields. ${ }^{c}$ The reaction was carried out in the dark.

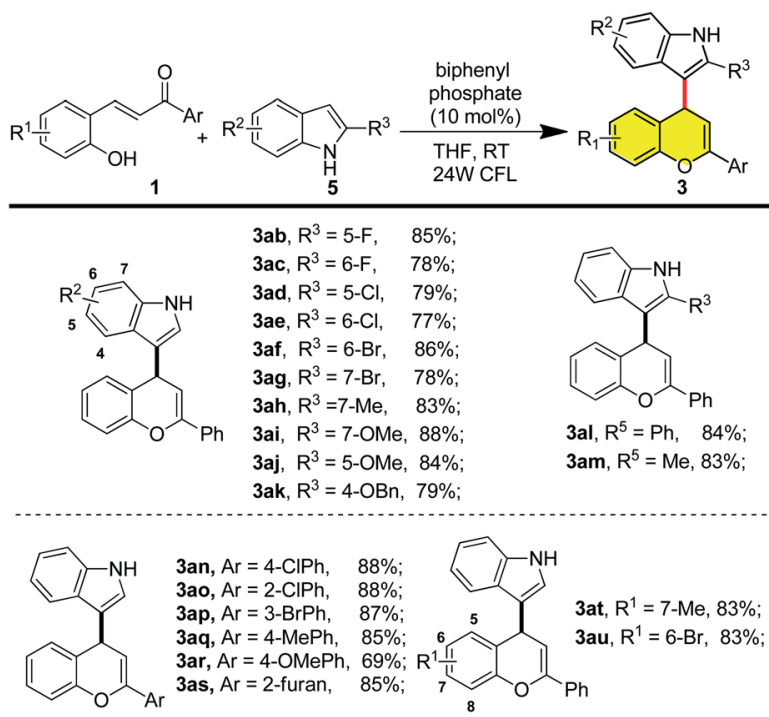

Scheme 1 Substrate scope with regard to indole as nucleophile.

gave comparable yield and showed better solubility for 2hydroxylchalcone 1a (Table 1, entry 9 and ESI†).

After establishing the optimal reaction conditions, the substrate scope was subsequently investigated. As shown in Scheme 1, electron-withdrawing group (e.g. $\mathrm{F}, \mathrm{Cl}$ and $\mathrm{Br}$ ) on the indole ring was compatible with the reaction conditions, providing corresponding products in good isolated yields (3ab to 3ag). Indoles with electron-donating groups (e.g. Me, methoxy) were also well tolerated, affording 3ah to 3ak in good yields. Substituents (e.g. phenyl and methyl) on the C-2 of indole did not affect the outcomes of this reaction (3al and 3am). Additionally, a variety of substituted 2-hydroxylchalcone was prepared and subjected to the standard reaction conditions. Satisfactorily, 2-hydroxylchalcones with electron-withdrawing groups $(\mathrm{Cl}, \mathrm{Br})$ and electron-donating group (Me, methoxy) could all be converted to hybrid flavonoids in good to excellent yields (3an to 3ar, 3at and 3au). Heterocycle such as furan was also compatible with the reaction conditions to afford flavonoid 3as in $85 \%$ yield.

Furthermore, this protocol was also applicable to other type of bifunctional nucleophiles to give rise to polycyclic flavonoids resembling diinsininol. As listed in Scheme 2, cyclohexa-1,3dione, phloroglucinol, 4-hydroxycoumarin could all engage in this type of reaction to give dioxabicyclo[3.3.1] nonane scaffold via tandem cycloisomerization/nucleophilic addition/ cyclization process. For those substrates, employment of $\mathrm{HCl}$ (for cyclohexa-1,3-dione and 4-hydroxycoumarin) or TsOH (for phloroglucinol) was more beneficial (see ESI $\dagger$ for details). When cyclohexa-1,3-dione was employed as nucleophile, various substituents on the aromatic ring of 2-hydroxyl chalcone were well compatible excepting the $\mathrm{Br}$ on the aromatic ring $\mathrm{A}$ (4ag), delivering the desired product in good to excellent yields (Scheme 2, 4aa to 4aj). Phloroglucinol was also a suitable nucleophile for this reaction, generating polycyclic flavonoids in acceptable yields. Different type of substitutes were tolerated on the aromatic ring B of 2-hydryxoychalcone (Scheme 2, 4 ba to 


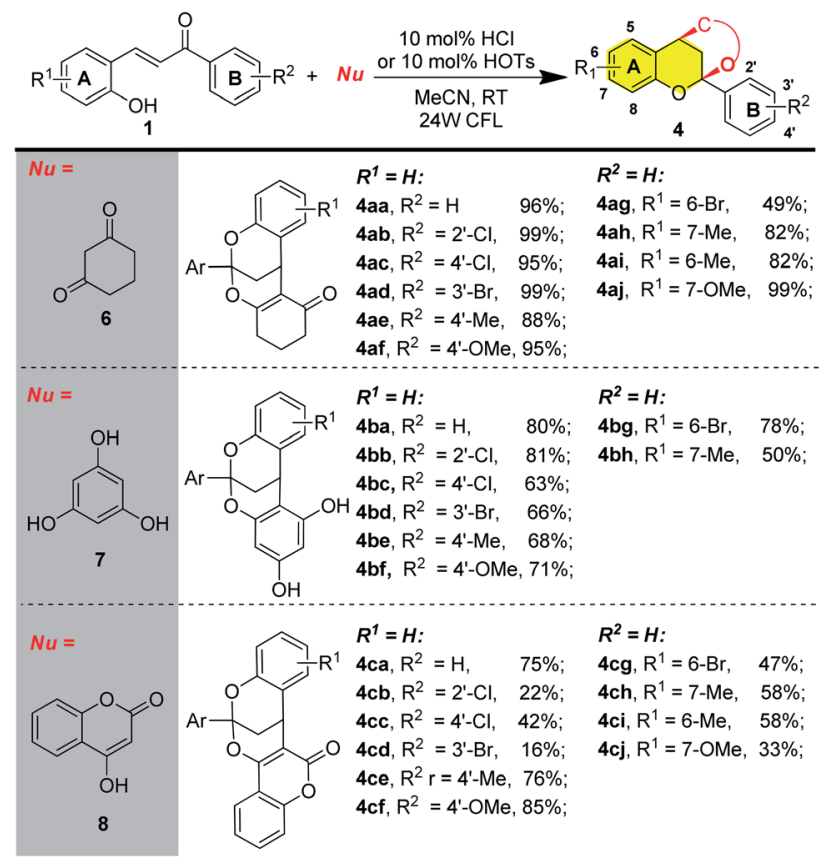

Scheme 2 Substrate scope using bifunctional nucleophiles.

$\mathbf{4 b g}$ ), while the electron-donating groups on the aromatic ring A were unfavorable for this reaction, resulting in much lower yields (Scheme 2, 4bh). Finally, 4-hydroxycoumarin was evaluated as the nucleophile to give access to coumarin hybrid flavonoids. For this specific substrate, the yields depended on the substituents on the aromatic rings of 2-hydryxoychalcone. Electron-withdrawing groups on the aromatic ring $\mathrm{B}$ of 2hydryxoychalcone were detrimental for reactions (4cb to $4 \mathbf{c d}$ ), affording much lower isolated yields than those with electrondonating substituents (4ce and 4cf) and substituents on the ring A were not well compatible with the reaction conditions (4cg to $4 \mathrm{cj})$.

To mimic the biosynthetic conditions, the sunlight-driven coupling of 2-hydroxychalcone with different nucleophiles was also carried out. As shown in Scheme 3, indole, cyclohexa-1,3dione, phloroglucinol and 4-hydroxycoumarin could all engage in the tandem process under the irradiation of sunlight using the same reaction conditions shown in Scheme 1 or 2, providing
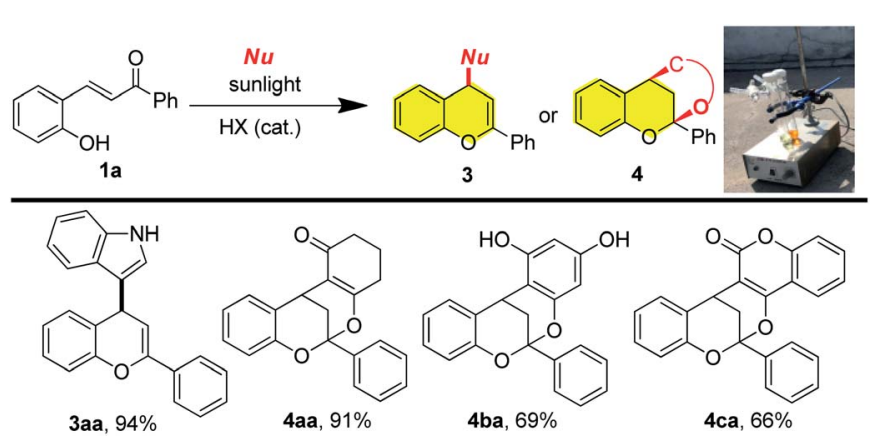

Scheme 3 Sunlight-driven synthesis of hybrid flavonoids. correspondent hybrid flavonoids 3aa to 4ca in comparable isolated yields with those using $24 \mathrm{~W}$ CFL as light source.

The enantioselective coupling of 2-hydroxychalcone was also explored using chiral phosphoric acid as organocatalyst via counter anion directed addition (Scheme 4). ${ }^{11}$ To our delight, flavonoid 3aa could be obtained in $99 \%$ yield with $45 \%$ ee employing catalytic $8 H$ - $(R)$-TRIP. Additionally, the enantioselective coupling of cyclohexa-1,3-dione to 2-hydroxy chalcone $\mathbf{1 a}$ could also be realized using $(R)$-TRIP as catalyst albeit in $67 \%$ yield with $27 \%$ ee. When phloroglucinol was employed reaction partner, flavonoid 4 ba could be obtained in 52\% yield with $70 \%$ ee using CPA1 as promoter (Scheme 4). Those initial results paved a new way for the enantioselective synthesis of hybrid flavonoids via counter-anion directed enantioselective addition. $^{\mathbf{1 1}}$

To gain in insights into the reaction pathway, a series of experimental were executed (Scheme 5). When the reaction was run in the dark, no product was detected with most of the starting material being recovered (Scheme 4, eqn (1)). The hemiketal 9 could be obtained in $62 \%$ yield when 2-hydroxylchalcone was irradiated by visible-light (Scheme 4, eqn (2)), which was in accordance with previous report. ${ }^{7}$ Treatment hemi-ketal 9 with stoichiometric amount of $\mathrm{HPF}_{6}$, flavylium salt 10 could be obtained in $62 \%$ yield (Scheme 4 , eqn (3)). Additionally, when presence of stoichiometric amount of $\mathrm{HPF}_{6}, 2$-hydroxyl chalcone could also be converted to flavylium salt 10 in $28 \%$ yield. The low yield was ascribed to the instability of flavylium cation (Scheme 4, eqn (4)). In sharp contrast, only trace of product could be observed when this reaction was run in the dark. We also found that addition of cyclohexa-1,3-dione 6 to flavylium 10 salt could smoothly proceeded in the dark, affording the flavonoid 4 aa in $75 \%$ yield (Scheme 4, eqn (5)). All these observations strongly supported the reaction proceeded through the tandem isomerization/cyclization/dehydration of 2hydroxychalcone to produce a transient flavylium ion, which was in situ captured by the nucleophile to afford hybrid flavonoids (Fig. 1).

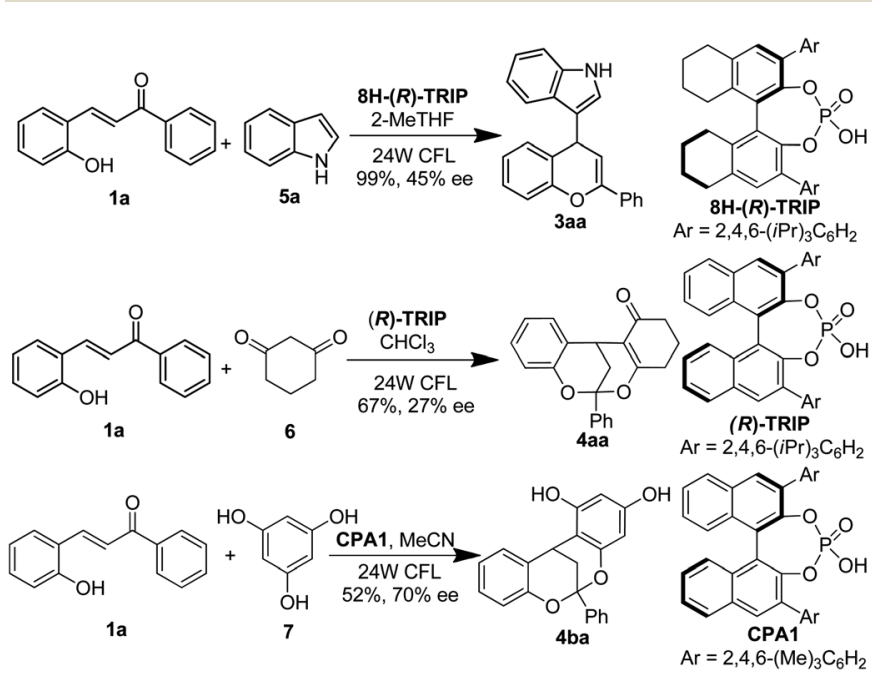

Scheme 4 Chiral phosphoric acid catalyzed enantioselective addition of nucleophile to 2-hydroxychalcone. 


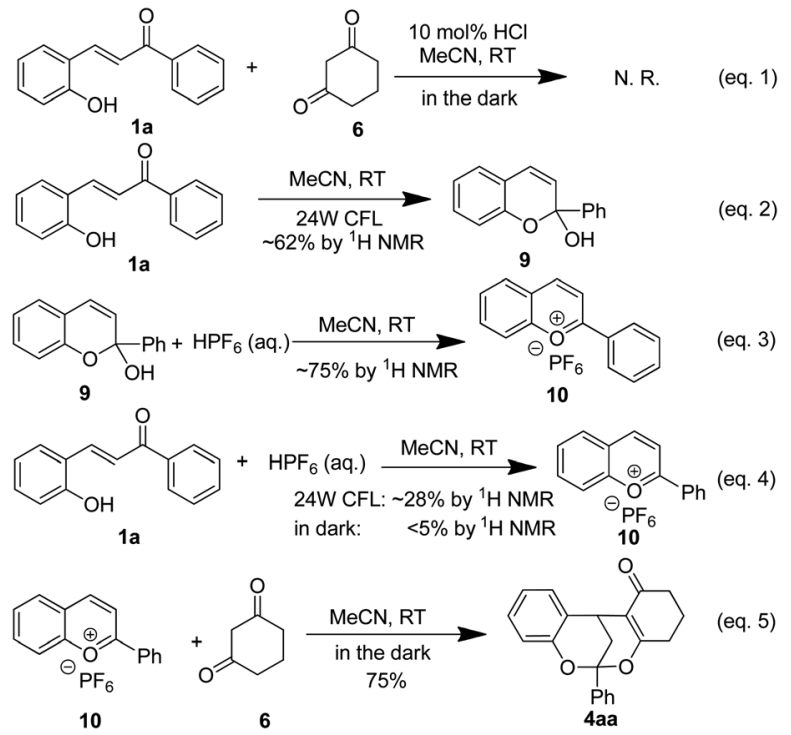

Scheme 5 Mechanistic studies.

\section{Conclusions}

In conclusion, a bio-inspired synthesis of hybrid flavonoids from 2-hydroxychalcone was developed. This protocol provided a facile entry to structurally diverse hybrid flavonoids under the irradiation of $24 \mathrm{~W}$ CFL. Based on the mechanistic studies, this reaction was proposed to proceed via the cascade double bond isomerization/dehydrated cyclization to afford a transient flavylium ion, which is in situ incepted by nucleophile. Furthermore, this protocol also provides novel strategy for the enantioselective synthesis of hybrid flavonoids.

\section{Conflicts of interest}

There are no conflicts to declare.

\section{Acknowledgements}

We are grateful for financial support from the National Natural Science Foundation of China (grants. 21722206, 21672171) and Shaanxi government. Financial support from Chinese Universities Scientific Fund and the Scientific Fund of Northwest A\&F University are also acknowledged.

\section{Notes and references}

1 (a) K. Suzuki, in Natural Products in Medicinal Chemistry V60, ed. S. Hanessian, Wiley-VCH, Verlag GmbH \& Co. KGaA, 2014; (b) K. Suzuki, Chem. Rec., 2010, 10, 291-307.

2 for review, see: (a) L. F. Tietze, H. P. Bell and S. Chandrasekhar, Angew. Chem., Int. Ed., 2003, 42, 39964028; for selected recent examples, see: $(b)$ K. Gademann, Chimia, 2006, 60, 841-845; (c) B. Dasari, R. Jimmidi and P. Arya, Eur. J. Med. Chem., 2015, 94, 497-508; (d) S. Christou, A. C. Edwards, S. Kaskun, R. G. Pritchard,
P. Quayle, Y. W. Song, I. J. Stratford, K. F. Williams and R. C. Whitehead, Tetrahedron, 2017, 73, 1295; (e) S. Choudhary, P. K. Singh, H. Verma, H. Singh and O. Silakari, Eur. J. Med. Chem., 2018, 151, 62-97.

3 (a) B. Harborne and T. J. Mabry, The Flavonoids: advances in research, Chapman and Hall, London, New York, 1982; (b) J. B. Harborne, The Flavonoids: advances in research since 1980, Chapman and Hall, London, New York, 1988; (c) B. Harborne, The Flavonoids: advances in research since 1986, Chapman \& Hall, London, New York, 1st edn, 1994; (d) C. A. Williams and R. J. Grayer, Nat. Prod. Rep., 2004, 21, 539-573; (e) .M. Andersen and K. R. Markham, Flavonoids: chemistry, biochemistry, and applications, CRC, Taylor \& Francis, Boca Raton, FL, 2006; ( $f$ ) K. Gould, K. M. Davies and C. Winefield, Anthocyanins: biosynthesis, functions, and applications, Springer, New York, 2009; $(\mathrm{g})$ S. Kumar and A. K. Pandey, Sci. World J., 2013, 2013, 16; (h) N. C. Veitch, Nat. Prod. Rep., 2013, 30, 988-1027.

4 (a) J. J. Vepsalainen, S. Auriola, M. Tukiainen, N. Ropponen and J. C. Callaway, Planta Med., 2005, 71, 1053-1057; (b) M. B. Calvert and J. Sperry, Chem. Commun., 2015, 51, 6202-6205.

5 (a) A. Ogundaini, M. Farah, P. Perera, G. Samuelsson and L. Bohlin, J. Nat. Prod., 1996, 59, 587-590; for the biomimetic synthesis, see: (b) C. Selenski and T. R. R. Pettus, Tetrahedron, 2006, 62, 5298-5307.

6 (a) L. A. Weston and U. Mathesius, J. Chem. Ecol., 2013, 39, 283-297; (b) B. Winkel-Shirley, Plant Physiol., 2001, 126, 485-493.

7 For selected reviews: (a) F. Pina, M. J. Melo, C. A. T. Laia, A. J. Parola and J. C. Lima, Chem. Soc. Rev., 2012, 41, 869908; (b) F. Pina, V. Petrov and C. A. T. Laia, Dyes Pigm., 2012, 92, 877-889; for selected examples: (c) L. Jurd, Tetrahedron, 1969, 25, 2367-2380; (d) D. Dewar and R. G. Sutherland, J. Chem. Soc. D, 1970, 272-273; (e) H. Hiroaki, Y. Akinobu, O. Tetsuo and H. Hiroshi, Bull. Chem. Soc. Jpn., 1999, 72, 2429-2435; (f) V. Petrov, A. M. Diniz, L. Cunha-Silva, A. J. Parola and F. Pina, $R S C$ Adv., 2013, 3, 10786-10794.

8 (a) F. Kröhnke and K. Dickoré, Chem. Ber., 1959, 92, 46-62;

(b) L. Jurd and B. J. Bergot, Tetrahedron, 1965, 21, 36973705; (c) L. Jurd and A. C. Waiss, Tetrahedron, 1965, 21, 1471-1483; (d) L. Jurd and R. Lundin, Tetrahedron, 1968, 24, 2653-2661; (e) G. Lee, K. Ishimaru, H. Iwasaki, K. Ohkata and K. Akiba, J. Org. Chem., 1991, 56, 2058-2066; $(f)$ G. A. Kraus, Y. Yuan and A. Kempema, Molecules, 2009, 14, 807-815; $(g)$ F. Benfatti, E. Benedetto and P. G. Cozzi, Chem.-Asian J., 2010, 5, 2047-2052; (h) Z. Y. Yang, Y. He and F. D. Toste, J. Am. Chem. Soc., 2016, 138, 9775-9778; (i) A. Alejo-Armijo, N. Glibota, M. P. Frías, J. Altarejos, A. Gálvez, S. Salido and E. Ortega-Morente, J. Agric. Food Chem., 2018, 66, 2151-2158.

9 C. Fichtner, G. Remennikov and H. Mayr, Eur. J. Org. Chem., 2001, 4451-4456.

10 (a) T. Nishikata, Y. Yamamoto and N. Miyaura, Adv. Synth. Catal., 2007, 349, 1759-1764; (b) G. Yin, L. Fan, T. Ren, C. Zheng, Q. Tao, A. Wu and N. She, Org. Biomol. Chem., 
2012, 10, 8877-8883; (c) N. C. Ganguly, P. Mondal and S. Roy, Tetrahedron Lett., 2013, 54, 2386-2390; (d) Y. Rao and G. D. Yin, Org. Biomol. Chem., 2013, 11, 6029-6035; (e) F. J. Wang, F. Chen, M. L. Qu, T. Li, Y. L. Liu and M. Shi, Chem. Commun., 2013, 49, 3360-3362; (f) Y. Rao, M. L. Liu, L. Wu and G. D. Yin, $R S C A d v$., 2014, 4, 64551-64558; $(g)$ C. Bingi, N. R. Emmadi, M. Chennapuram, Y. Poornachandra, C. G. Kumar, J. B. Nanubolu and K. Atmakur, Bioorg. Med. Chem. Lett., 2015, 25, 1915-1919; (h) A. Ganesan, J. Kothandapani and S. G. Subramaniapillai, RSC Adv., 2016, 6, 20582-20587; (i)
J. M. Guo, X. G. Bai, Q. L. Wang and Z. W. Bu, J. Org. Chem., 2018, 83, 3679-3687; (j) Y. N. Zhu, Z. G. Yao and F. Xu, Tetrahedron, 2018, 74, 4211-4219; (k) Y. S. Zhu, J. Guo, S. J. Jin, J. M. Guo, X. G. Bai, Q. L. Wang and Z. W. Bu, Org. Biomol. Chem., 2018, 16, 1751-1759; (l) J.-M. Guo, W.-B. Wang, J. Guo, Y.-S. Zhu, X.-G. Bai, S.-J. Jin, Q.-L. Wang and Z.-W. Bu, RSC Adv., 2018, 8, 15641-15651.

11 (a) R. J. Phipps, G. L. Hamilton and F. D. Toste, Nat. Chem., 2012, 4, 603-614; (b) M. Mahlau and B. List, Angew. Chem., Int. Ed., 2013, 52, 518-533. 